Techniques \& Culture

\title{
L'agriculture libre
}

Les outils agricoles à l'épreuve de l'open source

Quentin Chance et Morgan Meyer

\section{(2) OpenEdition}

Journals

Édition électronique

URL : https://journals.openedition.org/tc/8534

DOI : $10.4000 /$ tc. 8534

ISSN : 1952-420X

Éditeur

Éditions de l'EHESS

\section{Édition imprimée}

Date de publication : 6 juin 2017

Pagination : 236-239

ISBN : 978-2-7132-2707-3

ISSN : 0248-6016

Référence électronique

Quentin Chance et Morgan Meyer, "L'agriculture libre », Techniques \& Culture [En ligne], 67 | 2017, mis en ligne le 20 juin 2017, consulté le 29 septembre 2022. URL : http://journals.openedition.org/tc/8534 ; DOI : https://doi.org/10.4000/tc.8534 


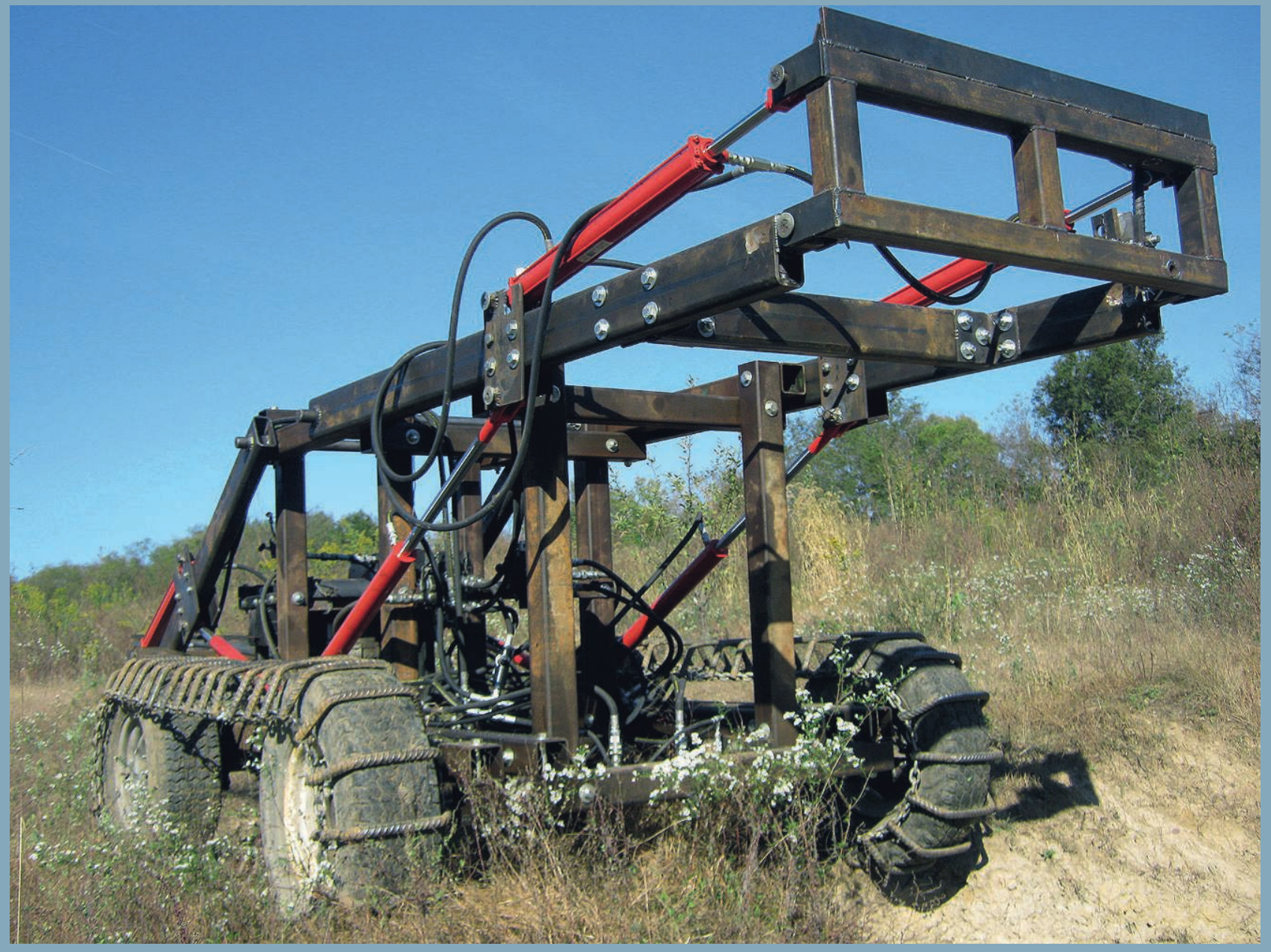




\section{I’agriculture libre}

\section{Les outils agricoles à l'épreuve de l'open source}

Les outils agricoles pourront-ils, à l'image des logiciels libres, devenir « libres » ? Les principes fondateurs de l'open source - la capacité de partager, distribuer, modifier et reproduire librement des informations - sont-ils transposables à l'agriculture? Si dans le domaine informatique, les logiciels libres sont maintenant une réalité, la question qui nous intéresse dans cet article consiste à savoir si des outils du secteur agricole comme des tracteurs, des graines ou des logiciels de gestion peuvent être créés et diffusés à travers des processus « libres », et si oui, comment.

Nous estimons qu'une différentiation et une description fine des outils agricoles s'imposent. On propose ainsi une typologie des outils mobilisés par l'agriculteur en fonction de leur matérialité :

- les outils mécaniques : ils regroupent la force motrice (traction motorisée ou animale) et les modules de travail liés (sur la terre ou les cultures);

- les outils du vivant : semences, intrants d'origine biologique et également ingénierie génétique;

- les outils numériques : outils de gestion, de suivi et de pilotage des exploitations.

Afin d'appréhender la façon dont se matérialise la démarche open source à travers les outils agricoles, procédons par étapes et, d'abord discutons de la question de l'open source dans le domaine agricole : son apparition, sa différence par rapport au modèle conventionnel propriétaire, son émergence dans d'autres secteurs (en réaction au modèle dominant plutôt privatif). Nous nous penchons ensuite sur deux cas d'étude : un logiciel de gestion intégré pour les exploitants agricoles développé par la société éponyme, Ekylibre; et une coopérative qui développe auprès des agriculteurs des techniques d'auto-construction d'outils agricoles, l'Atelier Paysan. Ces deux structures sont étudiées à travers leur histoire et leur forme d'organisation, la façon dont elles mettent en pratique les principes de l'open source, et en décrivant les outils fabriqués dans leur contexte économique et social. Les deux exemples nous permettent de considérer l'expression d'une même idée dans deux environnements 


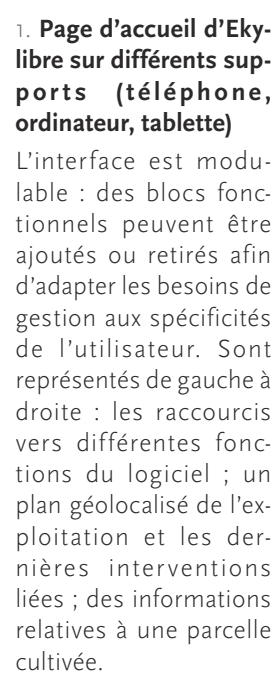

1. Page d'accueil d'Ekyports (téléphone ordinateur, tablette)

L'interface est modudes blocs foncajoutés ou retirés afin d'adapter les besoins de vers différentes fonctions du logiciel; un nières interventions liees : des informations cultivée. sociotechniques distincts. On observe d'une part un logiciel qui se situe «à la source » du mouvement informatique et d'autre part, un matériel physique qui s'élabore sur le terrain. Nous partons du constat que les deux structures mobilisent l'idée d'open source et nous faisons l'hypothèse que cette mise en contraste nous permettra de mieux saisir des façons de faire et des positionnements politiques. Les correspondances observées aident à discerner les spécificités de l'expression de l'open source au sein du monde agricole et nous invitent à nous intéresser à sa mise en œuvre.

Diverses méthodes ont été exploitées afin d'analyser les deux cas d'étude présentés ici. Des entretiens semi-structurés avec les fondateurs d'Ekylibre et de l'Atelier Paysan ont été réalisés d'avril à août 2015 et transcrits intégralement pour l'analyse. Simultanément, les différents documents et publications produits par les deux structures ont été examinés (rapports d'activité, sites web, brochures, forums, livres, etc.). Ces démarches ont été complétées par des observations et des visites de terrain.

Ekylibre et l'Atelier Paysan se ressemblent à plusieurs égards. Tous les deux se réfèrent au mouvement open source et s'investissent dans le partage des connaissances - des connaissances qui sont diffusés via des sites web, des démonstrations, des formations, des discussions via des forums. Les outils développés sont flexibles : après que la forme générique soit fabriquée ou téléchargée, l'outil est ensuite paramétré, adapté et configuré aux besoins spécifiques des utilisateurs. Tout en développant des outils, Ekylibre et l'Atelier Paysan développent aussi leur propre modèle économique, un modèle qu'ils disent devoir quasiment « inventer » eux-mêmes, car il se distingue des modèles classiques. Toutefois, en y regardant de plus près, on s'aperçoit que l'architecture, la matérialité et l'aspect financier des outils développés par Ekylibre et l'Atelier Paysan présentent des différences.

Larticle montre qu'il faut problématiser et détailler davantage en retraçant empiriquement comment l'open source se matérialise au cas par cas. Si on peut concevoir certains outils comme des

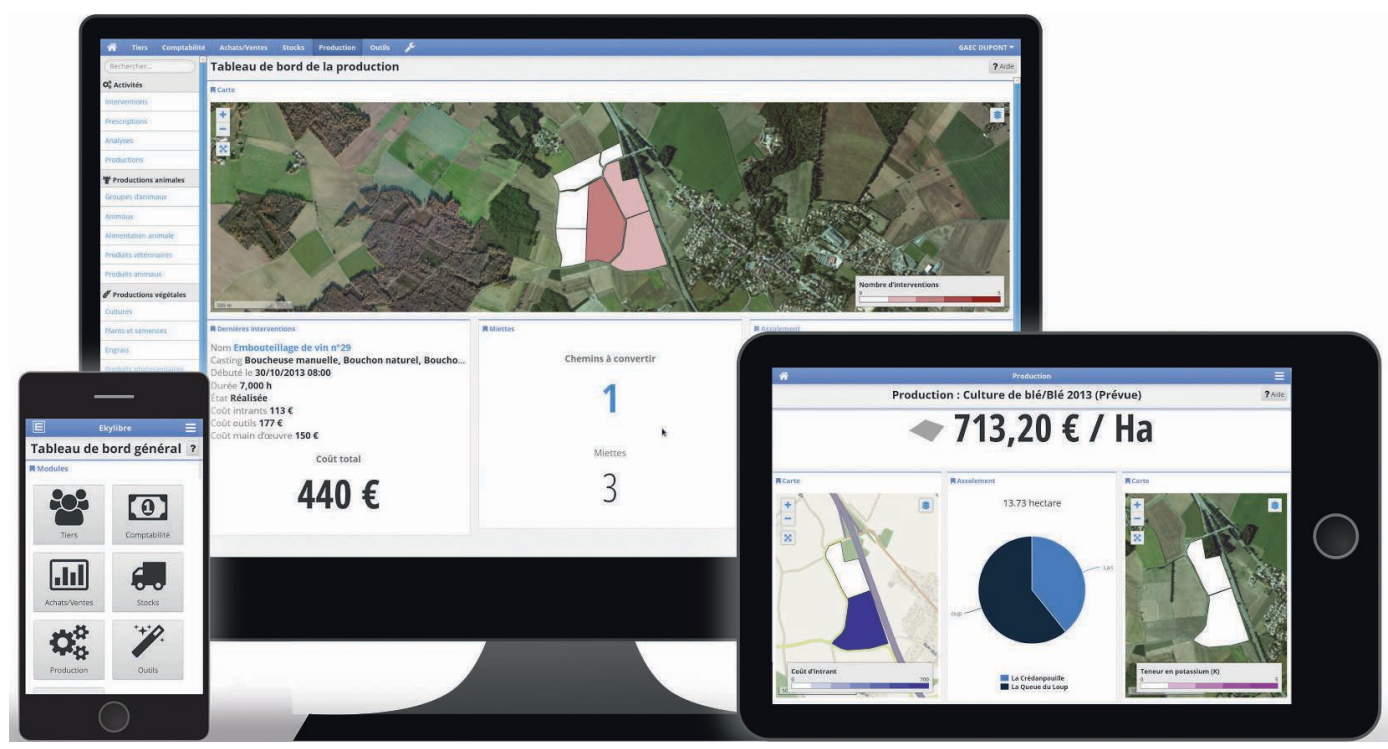


objets politiques, le terme politique ne renvoie pas à la même chose. Bien qu'Ekylibre et l'Atelier Paysan soient tous les deux liés à des communautés d'usagers, ces dernières sont organisées différemment et les échanges monétaires se font de façon dissemblable. Dans la pratique, l'idée d'open source ne se traduit pas seulement par des outils libres, partageables, collectifs, etc. Il est pour nous nécessaire d'examiner de quelle manière et jusqu'à quel degré des outils open source sont qualifiés comme tel et de préciser simultanément ce qu'on entend au cas par cas par « politique».

Il convient aussi de s'interroger sur le futur et le potentiel des outils agricoles open source. Les cas décrits dans ce texte sont récents et/ou émergents ; il sera utile de suivre leurs développement et trajectoires. Plusieurs questions se posent d'ores et déjà : à quelle échelle se diffuseront ces outils et ces projets? Resteront-elles nécessairement des initiatives de petite taille, plus ou moins locales ou nationales? Ou bien verrons-nous émerger des mouvements d'une envergure comparable à celle de Firefox dans le domaine informatique? La question des limites de l'agriculture libre - qu'elles soient géographiques, cognitives, matérielles ou économiques - se posera donc au fil des prochaines années.

\section{En ligne}

Retrouvez l'article complet sur revues.org, Techniques\&Culture 67 « Low tech? Wild tech! » : http://tc.revues.org.

\section{Les auteurs}

Quentin Chance est doctorant en sociologie à l'UMR Innovation à Montpellier (INRA, Montpellier SupAgro, CIRAD). Sa thèse porte sur les pratiques marchandes des agriculteurs biologiques, seuls à la ferme et sous forme collective dans les circuits longs, ainsi que sur leur rôle dans la construction des filières d'agriculture biologique. Originellement ingénieur en génie urbain, il s'est formé à la sociologie lors de son master à AgroParisTech. Il s'intéresse alors aux formes d'expression de l'open source en agriculture à l'UFR Sociologie d'AgroParisTech puis au LISIS.

Morgan Meyer est maître de conférences à AgroParisTech et chercheur au LISIS. Il détient un doctorat en sociologie et il a été postdoc au Département de Sociologie de l'Université de Sheffield et au Centre de Sociologie de l'Innovation à Mines ParisTech. Ses thèmes de recherche : l'émergence, la gouvernance et la mise en débat de nouvelles formes de biologie (biologie de synthèse, do-it-yourself biology) ; les lieux et pratiques du « knowledge brokering » (intermédiation des savoirs) et les frontières des sciences. Il participe actuellement au projet « L'innovation par les usages, un moteur pour l'agroécologie et les dynamiques rurales » (MCDR, 2015-2018).

\section{Iconographie}

Image d'ouverture. Le tracteur de LifeTrac est une machine open source qui pourrait coûter moitié moins 1. C) Ekylibre. https://ekylibre.com/press (Kit Visuel).

qu'un tracteur conventionnel ; et il est conçu pour durer toute une vie... Crédits : Open Source Ecology.

\section{Pour citer l'article}

Chance, Q. \& M. Meyer 2017 «L'agriculture libre. Les outils agricoles à l'épreuve de l'open source», Techniques\& Culture 67 «Low tech? Wild tech! », p. 236-239. 